\title{
Briófitas corticícolas de mata ciliar ao longo do Rio Uruguai, antes do alagamento da área pela Barragem de Itá, entre Santa Catarina e Rio Grande do Sul, Brasil
}

\author{
Denilson Fernandes Peralta ${ }^{1,3}$ e Francisco de Paula Athayde Filho ${ }^{2}$
}

Recebido: 03.04.2008; aceito: 04.09.2008

ABSTRACT - (Bark bryophytes of the riparian Forest along the Uruguai River, before flooding of the area by the dam of the electric power plan of Itá, between the states of Santa Catarina and Rio Grande do Sul in Brazil). The present study presents the bryophytes observed in phorophytes kept in the xylothec of the "Universidade do Vale do Rio dos Sinos" (UNISINOS). The samples were formerly collected inside the riparian forests along the Uruguai River, among the municipalities of Aratiba, Marcelino Ramos and Mariano Mouro (RS), and Corncórdia and Itá (SC), before the area was flooded because of the construction of the electric power plan of Itá. All bryophytes at the 187 phorophytes were analysed, totalizing 1,336 samples, resulting in the observation of 87 species, distributed in 53 genera and 31 families. The most rich families were Lejeuneaceae (13 species), Neckeraceae and Orthotrichaceae (six species).

Key-words: Brazilian South Region, bryophytes, Itá electric power plan, riparian forest

RESUMO - (Briófitas corticícolas da mata ciliar ao longo do Rio Uruguai, antes do alagamento da área pela Barragem de Itá, entre os estados de Santa Catarina e Rio Grande do Sul no Brasil). O presente estudo apresenta as briófitas observadas em forófitos depositados na xiloteca da Universidade do Vale do Rio dos Sinos (UNISINOS). As amostras foram originalmente coletadas no interior das matas ciliares ao longo do Rio Uruguai, entre os municípios de Aratiba, Marcelino Ramos e Mariano Mouro (RS), e Corncórdia e Itá (SC), antes que a área fosse alagada devido à construção da hidrelétrica de Itá. Todas as briófitas nos 187 forófitos foram analisadas, totalizando 1.336 amostras, resultando na observação de 87 espécies, distribuídas em 53 gêneros e 31 famílias. As famílias mais ricas foram Lejeuneaceae (13 espécies), Neckeraceae e Orthotrichaceae (seis espécies).

Palavras-chave: barragem de Itá, briófitas, mata ciliar, sul do Brasil

\section{Introdução}

A brioflora brasileira está representada por mais de 3.100 espécies e sua alta diversidade está relacionada principalmente às condições climáticas favoráveis ao seu estabelecimento (Yano 1996).

Para a região Sul do Brasil os trabalhos sobre briófitas foram realizados principalmente por autores gaúchos para no Estado do Rio Grande do Sul, sendo que Sehnem (1969, 1970, 1972, 1976, 1978, 1979, 1980) também cita espécimes do Estado de Santa Catarina. Os trabalhos desenvolvidos visaram estudos florísticos das briófitas sem enfoque ecológico.

Dessa maneira, para as Bryophyta (musgos), várias famílias, incluindo amostras de Santa Catarina, foram estudadas por Sehnem (1969, 1970, 1972, 1976, 1978, 1979, 1980) e a família Polytrichaceae por Farias (1987) apenas com amostras para o Rio Grande do Sul.

Para o Estado do Rio Grande do Sul as hepáticas (Marchantiophyta) tiveram várias famílias estudadas por Viana $(1970,1971,1974,1976,1981 \mathrm{a}, \mathrm{b}, \mathrm{c}, \mathrm{d}$, 1985, 1988, 1990, 1992, 1994a, b); Lorscheitter (1973) listou novas hepáticas folhoras; Lorscheitter (1977) estudou os gêneros de Lejeuneaceae, sem identificar espécies; Oliveira (1973) estudou as espécies de Radula; Lorscheitter-Baptista (1979) estudou hepáticas epífilas; Bueno (1984) estudou os gêneros de Jungermanniales, sem identificar espécies; Lemos-Michel (1983) estudou o gênero Frullania; Lemos-Michel \& Bueno (1992) estudaram o gênero Bazzania e Lemos-Michel (2001) publicou sua tese de doutorado com as briófitas epífitas sobre Araucaria angustifolia (Bert.) Kuntze.

1. Instituto de Botânica, Caixa Postal 3005, 01061-970 São Paulo, SP, Brasil

2. Universidade do Estado de Mato Grosso, Departamento de Ciências Biológicas, Campus de Nova Xavantina, BR-158, km 148, Caixa Postal 08, 78690-000 Nova Xavantina, MT, Brasil

3. Autor para correspondência: denilsonfp@yahoo.com.br 
Existem ainda os trabalhos que envolvem revisões brasileiras e citam amostras para os estados de Santa Catarina e Rio Grande do Sul, sem enfocar uma área específica.

Lemos-Michel \& Yano (1998) estudaram o gênero Bryopteris para o Brasil incluindo o Rio Grande do Sul e Bueno (1986) estudou o gênero Balantiopsis do Brasil.

Trabalhando com matas ciliares ou matas de galeria no Brasil são encontrados os trabalhos de Câmara \& Costa (2006) citando 19 espécies da Reserva Ecológica do IBGE, Distrito Federal e Genevro et al. (2006) que citam 49 espécies no Parque Municipal Mário Viana, Mato Grosso.

O trabalho que está sendo apresentado é o primeiro com briófitas de mata ciliar da região sul do Brasil.

No Brasil os trabalhos que tratam da relação entre briófitas e forófito foram os de Rebelo et al. (1995) estudando as comunidades de briófitas epífitas e relacionando as espécies com a poluição aérea em Santo André, Estado de São Paulo, neste trabalho a maioria das amostras foi identificada até gênero; o estudo realizado por Germano \& Pôrto $(1996,1998)$ que listaram 52 espécies de briófitas corticícolas em uma área de mata atlântica no Estado de Pernambuco e Lemos-Michel (2001) estudou as 38 espécies de hepáticas epifíticas sobre o forófito Araucaria angustifolia no Estado do Rio Grande do Sul.E, ainda, Visnadi (2004) analisou a distribuição das briófitas em diferentes fisionomias de cerrado caracterizando as espécies de acordo com o substrato.

Ainda não foram realizados estudos que descrevam o dano real das instalações humanas de grandes proporções nas comunidades vegetais. Especialmente com as briófitas não existem trabalhos inventariando áreas destinadas à inundação pela construção de usinas hidrelétricas.

As exsicatas de troncos (forófitos) referentes à comunidade arbórea existente antes da região ser alagada pela construção da Usina Hidroelétrica de Itá, continham as briófitas que existiam neste local e futuramente a listagem encontrada aqui pode ser utilizada para analisar o estado das espécies de briófitas epífitas da área.

Vista a importância de se conhecer melhor a flora das matas ciliares e, principalmente, das áreas cuja vegetação nativa tem sido destruída para a construção dos lagos das Usinas Hidrelétricas, decidiu-se realizar este trabalho, contribuindo para ampliar o conhecimento da brioflora brasileira.

\section{Material e métodos}

O Rio Uruguai e seus afluentes compreendem área total de $365.000 \mathrm{~km}^{2}$, antes de se juntarem ao rio Paraná e formarem o rio da Prata. Este possui $2.200 \mathrm{~km}$ de extensão e origina-se na confluência dos rios Pelotas e do Peixe, assumindo a direção Leste-Oeste, dividindo os Estados do Rio Grande do Sul e Santa Catarina. A área do rio Uruguai apresenta $73 \%$ de sua extensão no lado gaúcho e $27 \%$ no lado catarinense (MMA 2005). A bacia do Rio Uruguai abrange parcialmente dois dos Biomas brasileiros mais antropizados e fragmentados, que são a Mata Atlântica e os Pampas. No caso da Mata Atlântica, a bacia do Rio Uruguai apresenta fisionomias florestais e ecossistemas associados, como a Floresta Ombrófila Mista, Floresta Estacional Decidual, Floresta Estacional Semidecidual e campos naturais (MMA 2005). A região do alto rio Uruguai, onde o presente estudo foi executado, apresenta principalmente formações de Floresta Estacional Decidual e mata ciliar, mas outros tipos fisionômicos como Floresta Ombrofila Mista e Floresta Estacional Semidecidual também são observados na região do bioma Mata Atlântica (Leite \& Klein 1990).

Foram estudadas as briófitas encontradas nas exsicatas de forófitos depositados na xiloteca (PACA) da Universidade do Vale do Rio dos Sinos (UNISINOS). Os forófitos foram coletados durante a execução do projeto "Aproveitamento Científico do Lenho de Árvores Nativas do Estado do Rio Grande do Sul - Parte II - Bacia do Alto Rio Uruguai”, no período de março de 1994 a janeiro de 2000, em convênio com as Centrais Elétricas do Sul do Brasil (Eletrosul).

Durante a etapa de coleta dos forófitos, foram amostrados segmentos de $50 \mathrm{~cm}$ de comprimento do caule, raiz, ramos no interior das matas ciliares do alto rio Uruguai, entre os municípios de Aratiba, Marcelino Ramos e Mariano Mouro (RS) e Concórdia e Itá (SC) antes do alagamento da área para a construção da Barragem de Itá.

As briófitas foram herborizadas e depositadas no herbário PACA com duplicatas no herbário SJRP.

Não foram realizadas comparações com outros levantamentos porque a amostragem aqui ficou restrita aos $50 \mathrm{~cm}$ do caule dos forófitos.

O sistema de classificação adotado para a Bryophyta foi o de Buck \& Goffinet (2000) e, para Marchantiophyta, o de Crandall-Stotler \& Stotler (2000). A identificação foi baseada nos trabalhos 
de Florschütz (1964), Ochi (1980, 1981), Lisboa (1993), Sharp et al. (1994), Churchill \& Linares C. (1995), Buck (1998), Reese (1993), Gradstein (1994), Lemos-Michel \& Yano (1998), Oliveira-e-Silva \& Yano (2000), Lemos-Michel (2001) e Gradstein \& Costa (2003).

\section{Resultados e Discussão}

Foram estudadas 187 exsicatas de forófitos com 1.336 amostras (tabela 1). Foram identificadas 87 espécies de briófitas, distribuídas em 53 gêneros e 31 famílias (tabela 2). Deste total, 55 espécies pertencem à divisão Bryophyta (38 gêneros e 23 famílias) (figura 1) e 32 a Marchantiophyta (15 gêneros e oito famílias) (figura 2).

As famílias de Bryophyta mais ricas foram Neckeraceae e Orthotrichaceae, com seis espécies cada. Quatorze famílias apresentaram apenas um gênero e doze famílias, uma espécie. O gênero mais rico foi Fissidens, com cinco espécies (figura 1).

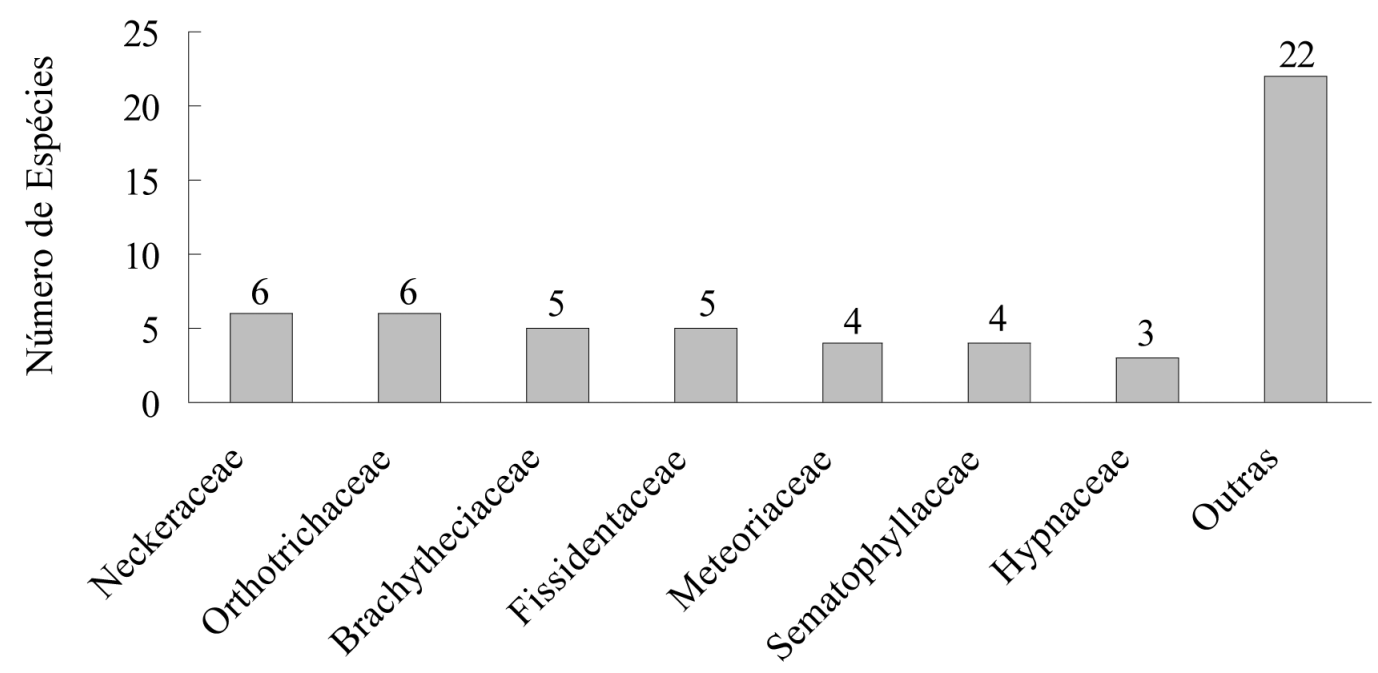

Figura 1. Número de espécies por família de Bryophyta encontrado na mata ciliar antes do alagamento pela Barragem de Itá. Outras = famílias representadas por no máximo duas espécies (17 famílias).

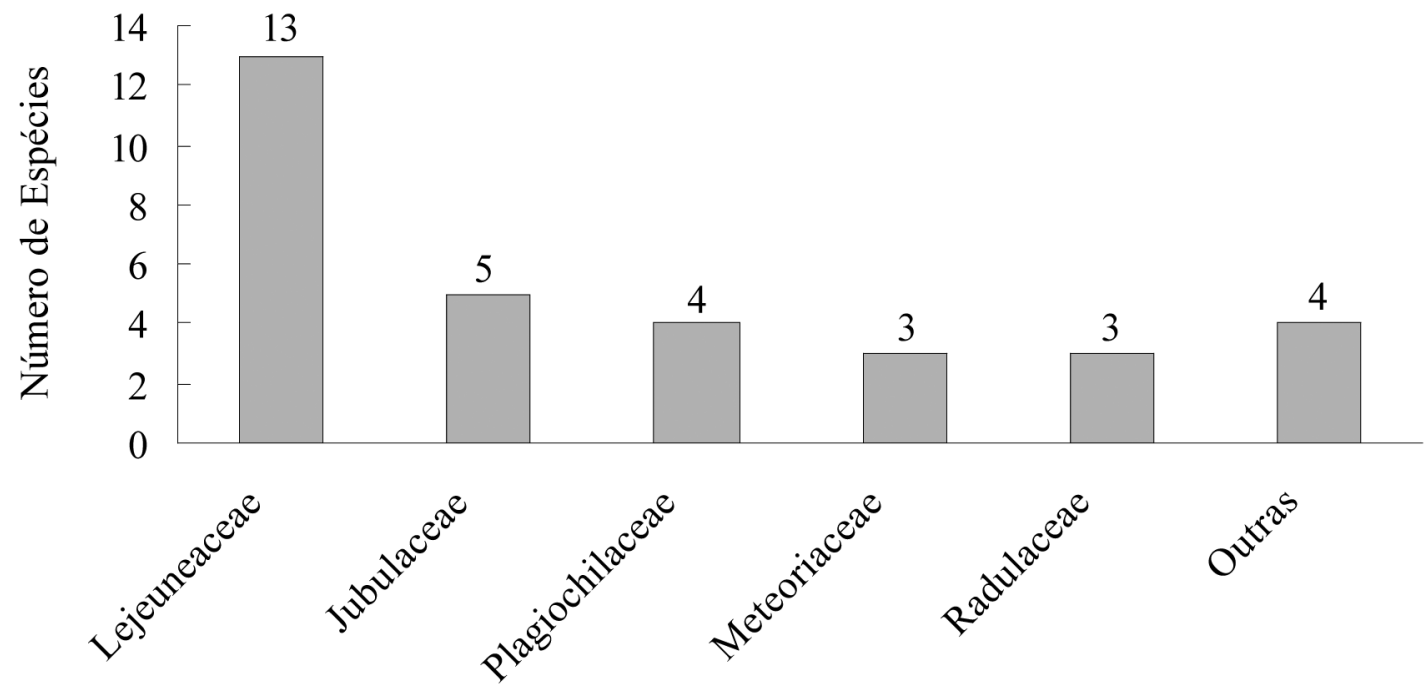

Figura 2. Número de espécies por família de Marchantiophyta encontrada na mata ciliar antes do alagamento pela Barragem de Itá. Outras = famílias representadas por no máximo duas espécies (três famílias). 
Tabela 1. Número e porcentagem de amostras de briófitas observados em exsicatas de forófitos, por município.

\begin{tabular}{lcccccc}
\hline \multirow{2}{*}{ Município } & \multirow{2}{*}{ Estado } & \multicolumn{2}{c}{ Amostras de briófitas } & & \multicolumn{2}{c}{ Exsicatas de forófitos } \\
\cline { 3 - 4 } & & $\mathrm{n}^{\mathrm{o}}$ & & & $\mathrm{n}^{\mathrm{o}}$ & $\%$ \\
\hline Aratiba & $\mathrm{RS}$ & 802 & 60 & & 106 & 57 \\
Marcelino Ramos & $\mathrm{RS}$ & 66 & 5 & & 3 & 2 \\
Mariano Moro & $\mathrm{RS}$ & 64 & 5 & & 51 & 27 \\
Concórdia & $\mathrm{SC}$ & 10 & 1 & & 13 & 7 \\
Itá & $\mathrm{SC}$ & 394 & 29 & & 14 & 7 \\
\hline Total & & $\mathbf{1 3 3 6}$ & $\mathbf{1 0 0}$ & & $\mathbf{1 8 7}$ & $\mathbf{1 0 0}$ \\
\hline
\end{tabular}

Dentre as Bryophyta estudadas, as espécies com freqüência de ocorrência superior a $20 \%$ sobre os forófitos analisados, em ordem decrescente, foram Helicodontium capillare (49,7\%), Papillaria nigrescens $(43,3 \%)$, Sematophyllum subpinnatum (38\%) e Neckera villae-ricae $(37,4 \%)$.

Com relação a Marchantiophyta, as famílias com maior riqueza específica foram Lejeuneaceae, com 13 espécies $(39,4 \%)$, Jubulaceae, com cinco $(15,2 \%)$ e Plagiochilaceae, com quatro $(12,1 \%)$. Sete famílias apresentaram apenas um gênero e duas famílias, uma única espécie. O gênero mais rico foi Frullania (Jubulaceae), com cinco espécies.

Entre as Marchantiophyta estudadas, aquelas espécies com freqüência de ocorrência superior a $20 \%$, em ordem decrescente, foram Frullania ericoides $(95,2 \%)$, Radula quadrata (80,8\%), Plagiochila corrugata $(49,7 \%)$ e Porella swartziana $(32,1 \%)$. Merece destaque a alta frequiência de ocorrência de Frullania ericoides em todas as áreas amostradas, indicando provavelmente maior grau de dispersão e tolerância a variações ambientais para ocupar muitos tipos de cascas dos vários forófitos analisados.

Por outro lado, várias espécies restringiram-se a uma amostra de forófito, como Rhynchostegium serrulatum, Fabronia ciliaris, Frullania caulisequa, Lophocolea bidentata (Geocalycaceae) de Marchantiophyta.

O elevado número de espécies de briófitas encontrado (87) fornece indícios de que esta área potencialmente teria uma riqueza muito maior, uma vez que não foram amostrados todo o restante dos troncos e copa dos forófitos e outros substratos como solo e rocha. Entretanto, devido à formação da barragem, hoje se torna difícil avaliar com precisão esta riqueza.

Quanto à distribuição das espécies de briófitas coletadas por município de ocorrência, constatou- se grande maioria em Aratiba (70 spp.), Mariano Mouro (59 spp.) espécies e Itá (26 spp.). Tal fato é justificável já que os dois primeiros concentraram a maior quantidade de forófitos amostrados com $57 \%$ e $27 \%$ respectivamente.

Apenas Papillaria nigrescens e Radula quadrata (2,2\% do total) foram observados nos cinco municípios amostrados, indicando provavelmente maior grau de dispersão e tolerância a variações ambientais; 13 espécies $(14,6 \%)$ ocorreram em quatro municípios, 15 espécies $(16,9 \%)$ em três; 23 espécies $(25,8 \%)$ em dois municípios e 36 espécies $(40,5 \%)$ em apenas um município (tabela 2).

Ariqueza de espécies de briófitas encontrada sobre apenas parte de um único tipo de substrato foi elevada quando comparada, por exemplo, com os dados obtidos nos trabalhos realizados em mata ciliar de Câmara $\&$ Costa (2006) que obtiveram 19 espécies (apenas Marchantiophyta e Antocerotophyta) e Genevro et al . (2006), 49 espécies. A mata deste estudo não existe mais, e assim é importante ressaltar a necessidade de outros inventários em áreas similares também sujeitas a inundações decorrentes da construção de hidrelétricas, para documentar a informação sobre a flora e fauna das matas ciliares que serão inundadas.

\section{Agradecimentos}

Os autores agradecem aos curadores dos herbários citados, pela cooperação, ao técnico responsável pela coleta das amostras de forófitos: Nádia Inês Jagmin, e aos colegas Ana Luisa Schneider, Carlos Rodrigo Lehn, Rosvita Bayer e Michele Cunha pelo auxílio durante as coletas do material briofítico das exsicatas de forófitos. E o apoio da Universidade do Vale do Rio dos Sinos (UNISINOS) e às Centrais Elétricas do Sul do Brasil (Eletrosul). 
Tabela 2. Distribuição nos municípios das briófitas das matas ciliares alagadas para a construção da Barragem de Itá. $1=$ Aratiba (RS); 2 = Marcelino Ramos (RS); 3 = Mariano Mouro (RS); 4 = Concórdia (SC); 5 = Itá (SC).

\begin{tabular}{|c|c|c|c|c|c|c|}
\hline \multirow[t]{2}{*}{ FAMÍLIAS } & \multirow[t]{2}{*}{ ESPÉCIES } & \multicolumn{5}{|c|}{ Municípios } \\
\hline & & 1 & 2 & 3 & 4 & 5 \\
\hline \multicolumn{7}{|c|}{ Bryophyta } \\
\hline \multirow[t]{5}{*}{ Brachytheciaceae } & Brachythecium occidentale (Hampe) A. Jaeger & $\mathbf{x}$ & & & & $\mathbf{x}$ \\
\hline & Rhynchostegium serrulatum (Hedw.) A. Jaeger & $\mathbf{x}$ & & & & \\
\hline & Squamidium nigricans (Hook.) Broth. & $\mathbf{x}$ & & $\mathbf{x}$ & & \\
\hline & Zelometeorium patulum (Hedw.) Manuel & $\mathbf{x}$ & & $\mathbf{x}$ & & $\mathbf{x}$ \\
\hline & Zelometeorium recurvifolium (Hornsch.) Manuel & $\mathbf{x}$ & & $\mathbf{x}$ & & \\
\hline \multirow[t]{2}{*}{ Bryaceae } & Brachymenium exile (Dozy \& Molk.) Bosch \& Sande Lac. & $\mathbf{x}$ & & $\mathbf{x}$ & & \\
\hline & Bryum limbatum Müll. Hal. & $\mathbf{x}$ & & & & \\
\hline Calymperaceae & Syrrhopodon parasiticus (Sw. ex Brid.) Paris & $\mathbf{x}$ & & $\mathbf{x}$ & & $\mathbf{x}$ \\
\hline \multirow[t]{2}{*}{ Cryphaeaceae } & Cryphaea filiformis (Hedw.) Brid. & $\mathbf{x}$ & & $\mathbf{x}$ & & $\mathbf{x}$ \\
\hline & Schoenobryum concavifolium (Griff.) Gangulee & $\mathbf{x}$ & & $\mathbf{x}$ & $\mathbf{x}$ & $\mathbf{x}$ \\
\hline Entodontaceae & Erythrodontium squarrosum (Hampe) Paris & $\mathbf{x}$ & $\mathbf{x}$ & $\mathbf{x}$ & & $\mathbf{x}$ \\
\hline \multirow[t]{2}{*}{ Erpodiaceae } & Aulacopilum glaucum Wilson & $\mathbf{x}$ & & & $\mathbf{x}$ & \\
\hline & Erpodium biseriatum (Austin) Austin & $\mathbf{x}$ & & & $\mathbf{x}$ & \\
\hline Fabroniaceae & Fabronia ciliaris (Brid.) Brid. & $\mathbf{x}$ & & & & \\
\hline \multirow[t]{5}{*}{ Fissidentaceae } & Fissidens diplodus Mitt. & $\mathbf{x}$ & & & & \\
\hline & Fissidens pellucidus Hornsch. & $\mathbf{x}$ & & & & \\
\hline & Fissidens prionodes Mont. & $\mathbf{x}$ & & & & \\
\hline & Fissidens radicans Mont. & $\mathbf{x}$ & & & & \\
\hline & Fissidens submarginatus Bruch & & & $\mathbf{x}$ & & \\
\hline Helicophyllaceae & Helicophyllum torquatum (Hook.) Brid. & $\mathbf{x}$ & & & & \\
\hline \multirow[t]{3}{*}{ Hypnaceae } & Isopterygium tenerifolium Mitt. & $\mathbf{x}$ & & $\mathbf{x}$ & & \\
\hline & Isopterygium tenerum (Sw.) Mitt. & $\mathbf{x}$ & & $\mathbf{x}$ & & \\
\hline & Taxiphyllum taxirameum (Mitt.) M. Fleisch. & $\mathbf{x}$ & & $\mathbf{x}$ & & $\mathbf{x}$ \\
\hline \multirow[t]{2}{*}{ Lembophyllaceae } & Orthostichella pentasticha (Brid.) W.R. Buck & $\mathbf{x}$ & & $\mathbf{x}$ & $\mathbf{x}$ & $\mathbf{x}$ \\
\hline & Pilotrichella flexilis (Hedw.) Ångström & $\mathbf{x}$ & & & & \\
\hline Leptodontaceae & Foorstroemia producta (Hornsch.) Par. & $\mathbf{x}$ & & $\mathbf{x}$ & & \\
\hline \multirow[t]{4}{*}{ Meteoriaceae } & Floribundaria flaccida (Mitt.) Broth. & & & & $\mathbf{x}$ & \\
\hline & Orthostichella pentasticha (Brid.) W.R. Buck & $\mathbf{x}$ & & $\mathbf{x}$ & $\mathbf{x}$ & $\mathbf{x}$ \\
\hline & Meteorium deppei (Hornsch. ex Müll. Hal.) Mitt. & $\mathbf{x}$ & & $\mathbf{x}$ & & $\mathbf{x}$ \\
\hline & Meteorium nigrescens (Sw. ex Hedw.) Dozy \& Molk. & $\mathbf{x}$ & $\mathbf{x}$ & $\mathbf{x}$ & $\mathbf{x}$ & $\mathbf{x}$ \\
\hline Mniaceae & Plagiomnium rhynchophorum (Harv.) T.J. Kop. & & & $\mathbf{x}$ & & \\
\hline Myriniaceae & Helicodontium capillare (Hedw.) A. Jaeger & $\mathbf{x}$ & & $\mathbf{x}$ & $\mathbf{x}$ & $\mathbf{x}$ \\
\hline \multirow[t]{6}{*}{ Neckeraceae } & Neckera ehrenbergii Müll. Hal. & & & $\mathbf{x}$ & & \\
\hline & Neckera villae-ricae Besch. & $\mathbf{x}$ & & $\mathbf{x}$ & $\mathbf{x}$ & $\mathbf{x}$ \\
\hline & Neckeropsis disticha (Hedw.) Kindb. & $\mathbf{x}$ & & & & \\
\hline & Neckeropsis undulata (Hedw.) Reichardt & $\mathbf{x}$ & & $\mathbf{x}$ & $\mathbf{x}$ & \\
\hline & Porotrichum longirostre (Hook.) Mitt. & $\mathbf{x}$ & & $\mathbf{x}$ & $\mathbf{x}$ & \\
\hline & Porotrichum substriatum (Hampe) Mitt. & & & $\mathbf{x}$ & & \\
\hline Orthotrichaceae & Macrocoma orthotrichoides (Raddi) Wijk \& Margad. & $\mathbf{x}$ & & $\mathbf{x}$ & & $\mathbf{x}$ \\
\hline
\end{tabular}




\begin{tabular}{|c|c|c|c|c|c|c|}
\hline \multirow[t]{2}{*}{ FAMÍLIAS } & \multirow[t]{2}{*}{ ESPÉCIES } & \multicolumn{5}{|c|}{ Municípios } \\
\hline & & 1 & 2 & 3 & 4 & 5 \\
\hline & Macromitrium cirrosum (Hedw.) Brid. & & & $\mathbf{x}$ & & \\
\hline & Macromitrium richardii Schwägr. & $\mathbf{x}$ & & $\mathbf{x}$ & & \\
\hline & Schlotheimia jamesonii (Arn.) Brid. & & & $\mathbf{x}$ & & \\
\hline & Schlotheimia rugifolia (Hook.) Schwägr. & $\mathbf{x}$ & & $\mathbf{x}$ & $\mathbf{x}$ & \\
\hline & Schlotheimia tecta Hook. f. \& Wilson & $\mathbf{x}$ & & & & \\
\hline Pilotrichaceae & Lepidopilum muelleri (Hampe) Spruce & $\mathbf{x}$ & & $\mathbf{x}$ & & \\
\hline Pottiaceae & Tortella tortuosa (Hedw.) Limpr. & & & $\mathbf{x}$ & & \\
\hline Pterobryaceae & Jaegerina scariosa (Lorentz) Arzeni & & & & $\mathbf{x}$ & \\
\hline \multirow[t]{5}{*}{ Racopilaceae } & Racopilum tomentosum (Hedw.) Brid. & $\mathbf{x}$ & & & & $\mathbf{x}$ \\
\hline & Sematophyllum adnatum (Michx.) E. Britton & $\mathbf{x}$ & $\mathbf{x}$ & $\mathbf{x}$ & $\mathbf{x}$ & \\
\hline & Sematophyllum galipense (Müll. Hal.) Mitt. & & & $\mathbf{x}$ & & \\
\hline & Sematophyllum subpinnatum (Brid.) E. Britton & $\mathbf{x}$ & & $\mathbf{x}$ & $\mathbf{x}$ & $\mathbf{x}$ \\
\hline & Sematophyllum subsimplex (Hedw.) Mitt. & $\mathbf{x}$ & & $\mathbf{x}$ & $\mathbf{x}$ & $\mathbf{x}$ \\
\hline \multirow[t]{2}{*}{ Stereophyllaceae } & Eulacophyllum cultelliforme (Sull.) W.R. Buck \& Ireland & $\mathbf{x}$ & & & & \\
\hline & Pilosium chlorophyllum (Hornsch.) Müll. Hal. & $\mathbf{x}$ & & & & \\
\hline Thuidiaceae & Thuidium tomentosum Schimp. & & & & $\mathbf{x}$ & \\
\hline TOTAL & & 43 & 3 & 35 & 17 & 17 \\
\hline \multicolumn{7}{|c|}{ Marchantiophyta } \\
\hline Bryopteridaceae & Bryopteris diffusa (Sw.) Nees & $\mathbf{x}$ & & & & \\
\hline Geocalycaceae & Lophocolea bidentata (L.) Dumort. & $\mathbf{x}$ & & & & \\
\hline \multirow[t]{5}{*}{ Jubulaceae } & Frullania arecae (Spreng.) Gottsche & $\mathbf{x}$ & & $\mathbf{x}$ & & \\
\hline & Frullania brasiliensis Raddi & $\mathbf{x}$ & & & & \\
\hline & Frullania caulisequa (Nees) Nees & & & $\mathbf{x}$ & & \\
\hline & Frullania ericoides (Nees ex Mart.) Mont. & $\mathbf{x}$ & & $\mathbf{x}$ & $\mathbf{x}$ & $\mathbf{x}$ \\
\hline & Frullania riojaneirensis (Raddi) Spruce & $\mathbf{x}$ & & $\mathbf{x}$ & & \\
\hline \multirow[t]{13}{*}{ Lejeuneaceae } & Acanthocoleus aberrans (Lindenb. \& Gottsche) Kruijt & $\mathbf{x}$ & & $\mathbf{x}$ & & \\
\hline & Ceratolejeunea cornuta (Lindenb.) Schiffner & & & $\mathbf{x}$ & & \\
\hline & Cheilolejeunea clausa (Nees \& Mont.) Steph. & $\mathbf{x}$ & & & & \\
\hline & $\begin{array}{l}\text { Cheilolejeunea discoidea (Lehm. \& Lindenb.) Kach. \& R.M. } \\
\text { Schust. }\end{array}$ & & & $\mathbf{x}$ & & \\
\hline & Cheilolejeunea rigidula (Mont.) R.M. Schust. & $\mathbf{x}$ & & & $\mathbf{x}$ & \\
\hline & Lejeunea flava (Sw.) Nees & $\mathbf{x}$ & & & $\mathbf{x}$ & \\
\hline & Lejeunea laetevirens Nees \& Mont. & $\mathbf{x}$ & $\mathbf{x}$ & $\mathbf{x}$ & & \\
\hline & Lejeunea maxonii (A. Evans) X.-L. He & & & $\mathbf{x}$ & & \\
\hline & Lejeunea phyllobola Nees \& Mont. ex Mont. & $\mathbf{x}$ & $\mathbf{x}$ & $\mathbf{x}$ & & $\mathbf{x}$ \\
\hline & Leucolejeunea unciloba (Lindenb.) A. Evans & $\mathbf{x}$ & $\mathbf{x}$ & $\mathbf{x}$ & & \\
\hline & Mastigolejeunea auriculata (Wilson \& Hook.) Schiffner & $\mathbf{x}$ & & & & \\
\hline & Microlejeunea epiphylla Bischler & $\mathbf{x}$ & & & & \\
\hline & Taxilejeunea obtusangula (Spruce) A. Evans & $\mathbf{x}$ & $\mathbf{x}$ & $\mathbf{x}$ & & \\
\hline \multirow[t]{3}{*}{ Metzgeriaceae } & Metzgeria albinea Spruce & $\mathbf{x}$ & & $\mathbf{x}$ & $\mathbf{x}$ & \\
\hline & Metzgeria convoluta Steph. & $\mathbf{x}$ & & $\mathbf{x}$ & & \\
\hline & Metzgeria hegewaldii Kuwah. & $\mathbf{x}$ & $\mathbf{x}$ & $\mathbf{x}$ & & $\mathbf{x}$ \\
\hline
\end{tabular}




\begin{tabular}{|c|c|c|c|c|c|c|}
\hline \multirow[t]{2}{*}{ FAMÍLIAS } & \multirow[t]{2}{*}{ ESPÉCIES } & \multicolumn{5}{|c|}{ Municípios } \\
\hline & & 1 & 2 & 3 & 4 & 5 \\
\hline \multirow[t]{4}{*}{ Plagiochilaceae } & Plagiochila adianthoides (Sw.) Lindenb. & $\mathbf{x}$ & & $\mathbf{X}$ & & \\
\hline & Plagiochila corrugata (Nees) Nees \& Mont. & $\mathbf{x}$ & & $\mathbf{x}$ & $\mathbf{x}$ & $\mathbf{x}$ \\
\hline & Plagiochila disticha (Lehm. \& Lindenb.) Lindenb. & & & $\mathbf{x}$ & & \\
\hline & Plagiochila rutilans Lindenb. & $\mathbf{x}$ & & $\mathbf{x}$ & & \\
\hline \multirow[t]{2}{*}{ Porellaceae } & Porella brasiliensis (Raddi) Schiffner & $\mathbf{x}$ & & $\mathbf{x}$ & & $\mathbf{x}$ \\
\hline & Porella swartziana (F. Weber) Trevis. & $\mathbf{x}$ & & $\mathbf{x}$ & $\mathbf{x}$ & $\mathbf{x}$ \\
\hline \multirow[t]{3}{*}{ Radulaceae } & Radula kegelii Gottsche ex Steph. & & & $\mathbf{x}$ & & $\mathbf{x}$ \\
\hline & Radula quadrata Gottsche & $\mathbf{x}$ & $\mathbf{x}$ & $\mathbf{x}$ & $\mathbf{x}$ & $\mathbf{x}$ \\
\hline & Radula tectiloba Steph. & $\mathbf{x}$ & & $\mathbf{x}$ & & $\mathbf{x}$ \\
\hline TOTAL & & 26 & 6 & 24 & 7 & 9 \\
\hline TOTAL GERAL & & 70 & 9 & 59 & 24 & 26 \\
\hline
\end{tabular}

\section{Literatura citada}

Buck, W.R. 1998. Pleurocarpous mosses of the West Indies. Memoirs of The New York Botanical Garden 82: $1-400$.

Buck, W.R. \& Goffinet, B. 2000. Morphology and classification of mosses. In: A.J. Shaw \& B. Goffinet (eds.). Bryophyte Biology. Cambridge University Press, New York, pp. 71-123.

Bueno, R.M. 1984. Gêneros de Jungermanniales (exc. Lejeuneaceae) no Rio Grande do Sul, Brasil. Dissertação de Mestrado. Universidade Federal do Rio Grande do Sul, Porto Alegre.

Câmara, P.E.A.S. \& Costa, D.P. 2006. Hepáticas e antóceros das matas de galeria da Reserva Ecológica do IBGE, RECOR, Distrito Federal, Brasil. Hoehnea 33: 79-87.

Churchill, S.P. \& Linares C., E.L. 1995. Prodromus Bryologiae Novo-Granatensis: Introduccion a la flora de musgos de Colombia. Biblioteca Jose Jeronimo Triana 12: 1-924.

Crandal-Stotler, B. \& Stotler, R. 2000. Morphology and classification of Marchantiophyta. In: A.J. Shaw \& B. Goffinet (eds.). Bryophyte Biology. Cambridge University Press, Cambridge, pp. 21-70.

Farias, H.C. 1987. A familia Polytrichaceae no Rio Grande do Sul, Brasil. Iheringia, série Botânica 32: 77-89.

Florschütz, P.A. 1964. The mosses of Suriname. Part 1. E.J. Brill, Leiden.

Genevro, J.A., Athayde Filho, F.P. \& Peralta, D.F. 2006. Briófitas de mata de galeria no Parque Municipal Mário Viana, Nova Xavantina, Mato Grosso, Brasil. Boletim do Instituto de Botânica 18: 149-157.

Germano, S.R. \& Pôrto, K.C. 1996. Floristic survey of epixilic bryophytes in relation remmant of the Atlantic forest (Timbaúba), PE, Brazil. 1. Hepaticopsida (except Lejeuneaceae) and Bryopsida. Tropical Bryology 12: 21-28.

Germano, S.R. \& Pôrto, K.C. 1998. Briófitas epíxilas de uma área remanescente de floresta atläntica (Timbaúba, PE, Brasil). 2. Lejeuneaceae. Acta Botanica Brasilica 12: 53-66.

Gradstein, S.R. 1994. Lejeuneaceae, Ptychantheae, Brachiolejeuneae. Flora Neotropica Monograph 62: 1-216.

Gradstein, S.R. \& Costa, D.P. 2003. The Hepaticae and Anthocerothae of Brazil. Memoirs of The New York Botanical Garden 87: 1-318.

Leite, P.F. \& Klein, R.M. 1990. Vegetação. In: O.V. Mesquita (ed.). Geografia do Brasil: Região Sul. v. 2. Instituto Brasileiro de Geografia e Estatística, Rio de Janeiro, pp.113-150.

Lemos-Michel, E. 1983. Frullania (Jungermanniales, Hepaticopsida) no Rio Grande do Sul, Brasil. Subgênero Diastoloba. Revista Brasileira de Botânica 6: 115123.

Lemos-Michel, E. 1999. Briófitas epífitas sobre Araucaria angustifolia (Bert.) Kuntze no Rio Grande do Sul, Brasil. Tese de Doutorado, Universidade de São Paulo, São Paulo.

Lemos-Michel, E. 2001. Hepáticas epifíticas sobre o pinheiro brasileiro no Rio Grande do Sul, Porto Alegre. Editora da Universidade Federal do Rio Grande do Sul, Porto Alegre.

Lemos-Michel, E. \& Bueno, R.M. 1992. O gênero Bazzania S.F. Gray (Hepaticae) no Rio Grande do Sul, Brasil. Hoehnea 19: 143-149.

Lisboa, R.C.L. 1993. Musgos acrocárpicos do Estado de Rondônia. Museu Paraense Emilio Goeldi, Belém.

Lorscheitter, M.L. 1973. Hepáticas folhosas primitivas, 
novas para o Rio Grande do Sul. Iheringia, série Botânica 17: 3-17.

Lorscheitter-Baptista, M.L. 1977. Flora Ilustrada do Rio Grande do Sul: Lejeuneaceae. Boletim do Instituto Central de Biociências, Botânica 36: 1-135.

Lorscheitter-Baptista, M.L. 1979. Notas sobre hepáticas epifilas do Rio Grande do Sul. Iheringia, série Botânica 24: $15-26$.

MMA. 2005. Termo de referência para o estudo de avaliação ambiental integrada dos aproveitamentos hidrelétricos na bacia do rio Uruguai. Secretaria de Qualidade Ambiental nos Assentamentos Humanos, Ministério do Meio Ambiente, Brasília.

Ochi, H. 1980. A revision of the Neotropical Bryoideae, Musci (first part). The Journal of the Faculty of Education Tottori University, Natural Science 29: 49-154.

Ochi, H. 1980. A revision of the Neotropical Bryoideae, Musci (second part). The Journal of the Faculty of Education Tottori University, Natural Science 30: 2155.

Oliveira-e-Silva, M.I.M.N. \& Yano, O. 2000. Musgos de Mangaratiba e Angra dos Reis, Rio de Janeiro, Brasil. Boletim do Instituto de Botânica 14: 1-137

Oliveira,P.L. 1973. Espécies do gênero Radula Dumortier ocorrentes no Rio Grande do Sul, Brasil (Hepáticas). Iheringia, série Botânica 18: 48-53.

Rebelo, C.F., Stuffaldi-de-Vuono, Y. \& Domingos, M. 1995. Estudo ecológico de comunidades de briófitas na Reserva Biológica de Paranapiacaba, SP, em trechos de floresta sujeitos à influência da poluição aérea. Revista Brasileira de Botânica 18: 1-15.

Reese, W.D. 1993. Calymperaceae. Flora Neotropica Monograph 58: 1-102.

Sehnem, A. 1969. Musgos sul-brasileiros. I. Pesquisas, Botânica 27: 1-41.

Sehnem, A. 1970. Musgos sul-brasileiros. II. Pesquisas, Botânica 28: 1-117.

Sehnem, A. 1972. Musgos sul-brasileiros. III. Pesquisas, Botânica 29: 1-70.

Sehnem, A. 1976. Musgos sul-brasileiros. IV. Pesquisas, Botânica 30: 1-79.

Sehnem, A. 1978. Musgos sul-brasileiros. V. Pesquisas, Botânica 32: 1-170.

Sehnem, A. 1979. Musgos sul-brasileiros. VI. Pesquisas, Botânica 33: 1-149.

Sehnem, A. 1980. Musgos sul-brasileiros. VII. Pesquisas, Botânica 34: 1-121.

Sharp, A.J., Crum, H. \& Eckel, P.M. 1994. The moss flora of Mexico. Memoirs of The New York Botanical Garden 69: 1-1113.

Viana, E.C. 1970. Marchantiales e Anthocerotales coletadas no Rio Grande do Sul. Iheringia, série Botânica 14: 45-54.
Viana, E.C. 1971. Considerações sobre algumas hepáticas de Gramado, no Rio Grande do Sul, Brasil. Iheringia, série Botânica 15: 3-18.

Viana, E.C. 1974. Ramificações masculinas, femininas e esporogônio de Telaranea sejuncta var. breviseta (Herz.) Fulford (Hepaticopsida). Iheringia, série Botânica 19: 3-10.

Viana,E.C. 1976. Marchantiales (Hepaticopsida) coletadas no Rio Grande do Sul. Tese de Livre Docência, Universidade Federal do Rio Grande do Sul, Porto Alegre.

Viana,E.C. 1981a. Nota sobre a ocorrência de brotações em anteridióforos de Marchantia chanopoda L.(Hepaticae). Iheringia, série Botânica 28: 43-46.

Viana, E.C. 1981b. O gênero Riccia (Marchantiales) no Rio Grande do Sul, Brasil, I. Subg. Ricciella e Tallocarpus. Rickia 9: 71-80.

Viana,E.C. 1981c. Sobre a ocorrência de Monoclea Hook. (Hepaticae) no Rio Grande do Sul, Brasil. Iheringia, série Botânica 26: 165-167.

Viana,E.C. 1981d. Sphaerocarpus muccilloi, a new hepatic from Brazil. Lindbergia 7: 58-60.

Viana, E.C. 1985. Flora Ilustrada do Rio Grande do Sul: Marchantiales. Boletim do Instituto Central de Biociências, Botânica 38: 1-213.

Viana, E.C. 1988. Donnes additionnelles sur Riccia jovetastiae E.C. Vianna, espece bresilienne (Rio Grande do Sul). Cryptogamie, Bryologie et Lichénologie 9: 73-75.

Viana,E.C. 1990. Dados adicionais sobre Riccia fruchartii Steph. Iheringia, série Botânica 42: 105-107.

Viana,E.C. 1992. Brotações nos talos femininos de Riccia paraguayensis Spruce. Iheringia, série Botânica 42: 105-107.

Viana, E.C. 1994a. Variações morfológicas em Riccia brasiliensis Schiffner (Ricciaceae) no Rio Grande do Sul. Iheringia, série Botânica 44: 147-151.

Viana, E.C. 1994b. Variações morfológicas em Riccia lamellosa Raddi (Ricciaceae) no Rio Grande do Sul. Iheringia, série Botânica 4: 153-157.

Visnadi, S.R. 2004. Distribuição da brioflora em diferentes fisionomias do cerrado da Reserva Biológica da Estação Experimental de Mogi-Guaçu, SP, Brasil. Acta Botanica Brasilica 18: 965-973.

Yano, O. 1996. A checklist of the Brazilian bryophytes. Boletim do Instituto de Botânica 10: 47-232. 